\title{
Temporally varying larval settlement, competition, and coexistence in a sessile invertebrate community
}

\author{
Kyle F. Edwards ${ }^{1,2, *}$, John J. Stachowicz ${ }^{1}$ \\ ${ }^{1}$ Center for Population Biology, University of California Davis, Davis, California 95616, USA \\ ${ }^{2}$ Present address: Kellogg Biological Station, Michigan State University, Hickory Corners, Michigan 49060, USA
}

\begin{abstract}
Storage effect theory describes conditions under which recruitment fluctuation can promote the coexistence of multiple species competing for a single resource. In communities of benthic marine animals, larval settlement is often highly variable, and it has long been suspected that settlement variability promotes coexistence via the storage effect, but no empirical studies have provided quantitative support for this. Here we tested for a necessary component of the storage effect using the bryozoan Watersipora subtorquata, a poor competitor during recruitment. We found that from 2008 to 2009, per capita settlement of W. subtorquata was uncorrelated with the strength of interspecific competition during recruitment; this implies that the storage effect has the potential to promote the persistence of this species. We also found a difference between years in patterns of within-year fluctuation. In 2008, settlement and competition were uncorrelated, while in 2009, settlement and competition were strongly positively correlated, minimizing any role for the storage effect within 2009. The difference between years is due to reduced settlement, in 2009, of a dominant competitor whose settlement phenology differs from that of Watersipora. Our results add to supporting evidence for the storage effect from terrestrial plants and zooplankton, while suggesting that the strength of this mechanism may itself be temporally variable.
\end{abstract}

KEY WORDS: Storage effect · Bryozoan · Supply-side ecology · Larval settlement · Recruitment · Fluctuation-dependent coexistence

Resale or republication not permitted without written consent of the publisher

\section{INTRODUCTION}

Settlement that varies over time is a defining characteristic of benthic marine communities. Most species reproduce via pelagic larvae or spores that are produced or released at temporally varying rates, and this variation in production is compounded by spatio-temporal variation in oceanographic processes, resulting in settlement that can be highly variable for both animals and plants (Caley et al. 1996, Underwood \& Keough 2000). Population dynamics (Gaines \& Roughgarden 1985) and the strength of interactions such as predation and facilitation (Berlow 1999) can vary as a result of settlement rates.
However, we have little understanding of how variation in settlement over time affects the maintenance of diversity in benthic communities.

Storage effect theory has shown in general terms how temporal variation in the environment can maintain species diversity, if competing species have different responses to varying conditions (Chesson \& Warner 1981, Chesson 2008). We can describe the potential operation of this mechanism in benthic communities by considering a system in which variable recruitment is driven by variable settlement, and in which newly settled individuals compete for space or some spatially localized resource. The first requirement for the storage effect is that competing 
species have species-specific responses to environmental variation (Chesson 2008). One example of a species-specific response is a distinct pattern of per capita settlement rate (Warner \& Chesson 1985). If different species have different settlement patterns, then it is possible for variable settlement to increase the importance of intraspecific competition, relative to interspecific competition, during recruitment. This relative increase in intraspecific competition is the hallmark of stable coexistence mechanisms (Chesson 2008). The second requirement of the storage effect is buffered population growth (Chesson 2008); this means that some features of the organism's life history allow its population to make gains during favorable periods, while not having these gains completely erased during unfavorable periods. In the context of benthic organisms, buffered population growth is likely if the organism is most sensitive to competition during recruitment, while individuals post-recruitment are relatively long-lived and resistant to competition. This means that, for a specific population, even if interspecific competition experienced by juveniles is currently very strong, the older stages of the population will not suffer greatly, thereby buffering the population against intense competition at the recruitment stage.

The third requirement for the storage effect pertains to how species-specific settlement patterns affect the density-dependence of competition. When a species is at high density in the community, its periods of peak settlement will result in strong intraspecific competition, due to high settler density of conspecifics; over time, this leads to a positive covariance between a species' per capita settlement rate and the total strength of competition during recruitment. In other words, the periods when a species could potentially make the greatest population gains are also the periods when it experiences the most competition. In contrast, when a species is at low density, variation in competition during recruitment will be driven by variation in the settlement of interspecific competitors. If a species' pattern of per capita settlement differs from the patterns of its competitors, then periods of peak settlement may differ from periods of peak competition. This may result in a weak or negative covariance between a species' per capita settlement rate and the total strength of competition during recruitment. If that species has buffered population growth, it will be able to make population gains during periods when settlement is high but competition is weak, while persisting during periods when competition is strong. Therefore, the fact that the covariance between settlement and competition decreases as a species becomes rarer is the third essential ingredient for the promotion of coexistence by the storage effect (Chesson 2008).

Early work on the storage effect was motivated by the variability in larval recruitment that is typical of benthic marine communities (Sale 1977, Chesson \& Warner 1981, Warner \& Chesson 1985), but following the development of the theory there have been no empirical applications of this mechanism to benthic communities. Thus far, evidence for this mechanism has come from terrestrial plants (desert annuals: Pake \& Venable 1995, Angert et al. 2009; tropical trees: Kelly \& Bowler 2002; temperate grasses: Adler et al. 2006) and freshwater zooplankton (Cáceres 1997). How can we test whether the storage effect promotes coexistence in a benthic community? Direct experimental evidence that settlement fluctuation promotes coexistence would require experimental manipulation of the variance in settlement, over many generations in a closed system, in order to test whether species diversity is reduced when settlement variability is reduced. This approach is infeasible for most communities, especially benthic marine communities where the scale of population regulation can be large. An alternative approach is to use time series data on settlement and competition, in combination with theory, to estimate the effect of fluctuating settlement on the population dynamics of competing species. Fully quantifying the storage effect using this approach would require information on competitive interactions during recruitment for all of the most important competitors in the community (e.g. Adler et al. 2006, Chesson 2008, Angert et al. 2009)

The approach we took here uses observational and experimental times series data to quantify a necessary, but not sufficient, component of the storage effect for a sessile colonial invertebrate, the bryozoan Watersipora subtorquata. We consider this an important first step towards testing the storage effect in benthic communities. The focal process is competition for patches of free space among the individuals that settle into those patches. The aim was to quantify, for $W$. subtorquata, the relationship between its temporal pattern of larval settlement and the temporal pattern of interspecific competition experienced by young $W$. subtorquata colonies. This is a necessary component of the storage effect, because in this community, the storage effect can only benefit $W$. subtorquata if its pattern of settlement is uncorrelated or negatively correlated with interspecific competition during recruitment; our rationale for this approach is described further in 'Materials and methods'. 


\section{MATERIALS AND METHODS}

\section{Background}

This study was performed in the fouling community at Spud Point Marina, Bodega Harbor, California, USA $\left(38^{\circ} \mathrm{N}, 123^{\circ} \mathrm{W}\right)$. The sessile, epibenthic invertebrate community includes bryozoans, ascidians, sponges, anemones, mussels, barnacles, polychaetes, and hydroids. Larvae of these organisms settle into patches of unoccupied space, and upon metamorphosis attempt to grow into that space. In the process, newly settled individuals compete with other newly settled individuals, as well as older individuals bordering a patch. Here we focused on competition between newly settled individuals, as this interaction has the potential to be greatly affected by temporal variation in larval production and settlement.

The focal species in this study is the crustose bryozoan Watersipora subtorquata. Prior work has shown that small $W$. subtorquata colonies are poor competitors for several weeks after settlement. For example, in one 4 wk observation period, $\sim 80 \%$ of colonies smaller than $10 \mathrm{~mm}^{2}$ were rapidly overgrown after contacting a heterospecific (Edwards \& Stachowicz 2011; initial colony size is typically $\sim 0.2 \mathrm{~mm}^{2}$, and a size of $10 \mathrm{~mm}^{2}$ is reached at $\sim 4 \mathrm{wk}$ of age). However, larger colonies are more successful in overgrowth competition, and among the 6 common crustose colonial species in this community, $W$. subtorquata has the second greatest competitive ability (quantified using interactions among colonies $>1 \mathrm{~cm}^{2}$; Edwards \& Stachowicz 2010). Furthermore, W. subtorquata colonies have the potential to be long-lived, relative to the temporal scale over which settlement fluctuates ( 2 to $4 \mathrm{wk}_{\text {; }}$ see 'Field methods'). Mortality from causes other than overgrowth appears to be dominated by senescence, and senescence results in partial mortality for $W$. subtorquata, with older parts of the colony senescing while younger parts continue to grow. Field observations indicate that the proportion of the colony that senesces is $\sim 0.002 \mathrm{~d}^{-1}$ (Edwards \& Stachowicz 2010), and that colonies may persist for $>1 \mathrm{yr}$ (K. Edwards pers. obs.). Because W. subtorquata is sensitive to competition at small sizes, but is a strong competitor at large sizes and is relatively long-lived, settlement fluctuation has the potential to benefit this species' persistence via the storage effect.

In this study, we focused primarily on the effects of intra-annual, rather than interannual, variation in larval settlement. This scale is appropriate for this community because reproduction, growth, and mortality occur continuously throughout the year; most of the common species live less than 1 yr (Edwards \& Stachowicz 2010); settlement rates can change greatly over several weeks (Edwards \& Stachowicz 2010); and growth is fast enough that newly opened patches are filled within 5 to $8 \mathrm{wk}$ (K. Edwards pers. obs.; see 'Field methods'). For Watersipora in particular, reproductive maturity can occur at 3 to $5 \mathrm{wk}$ (S. Hart pers. comm.), although at some sites, 7 to 9 wk is more common (Marshall \& Keough 2009), and therefore the period of high reproduction from spring to fall may include 6 to 8 new generations. Accordingly, we present analyses that encompass both years, as well as analyses for each year individually. Dividing the time series by calendar year is sensible, because peak settlement occurs from April to November and is minimal during the winter.

\section{Field methods}

Larval production in the fouling community occurs largely from late spring through the fall, and different species often exhibit different phenologies within this period (Edwards \& Stachowicz 2010). We used a time series of recruitment experiments to quantify variation in settlement and the strength of competition during this period, for 2 consecutive years. Because settlement rate can change greatly over the course of several weeks, we deployed a new recruitment experiment approximately every $3 \mathrm{wk}$ (hereafter referred to as 'temporal replicates'). This resulted in 10 temporal replicates over the course of the settlement season, in each year. For each replicate, we deployed 16 PVC plates (each $10 \times 10 \mathrm{~cm}$ ), within $2 \mathrm{~m}$ of each other, face down at $1 \mathrm{~m}$ depth. Plates were taken to the lab at $3 \mathrm{wk}$ to count newly settled individuals (hereafter 'settlers'). From half of the plates, all organisms except Watersipora subtorquata were removed, and these plates were continually weeded (every $2 \mathrm{wk}$ ) to maintain monocultures. Plates were returned to the marina after counting, and final abundance (percent cover) was taken at $14 \mathrm{wk}$, at which point surviving individuals are large and either sexually mature or near sexual maturity. Final abundances and the number of settlers present at $3 \mathrm{wk}$ were used to estimate the strength of competition and settlement rates over time. Hereafter we refer to individuals at $14 \mathrm{wk}$ as 'recruits,' and we describe their survival and growth during this period as 'recruitment,' as distinct from settlement.

Settlement occurs continuously in this system, and therefore individuals settling into patches of free space will be competing with other individuals of a 
range of ages. Newly deployed substrates reach $100 \%$ cover within 5 to $8 \mathrm{wk}_{\text {; }}$ therefore, individuals surviving to recruitment (14 wk) will almost surely have settled before 5 to $8 \mathrm{wk}$. However, because individuals grow exponentially at small size (Edwards \& Stachowicz 2010), and early survival is sizedependent (Edwards \& Stachowicz 2011), the probability of surviving to recruitment declines strongly for later-arriving settlers. We therefore considered recruitment experiments deployed $3 \mathrm{wk}$ apart to be approximately independent, in the sense that there was little overlap between them in the temporal pools of settlers that may become successful recruits. Likewise, we used the number of settlers present by $3 \mathrm{wk}$ in a replicate to represent the settlement rate that is relevant for competition during recruitment, in that replicate.

\section{Quantifying settlement and competition}

In order to quantify settlement rate on a per capita basis, we used long-term panels (deployed >3 yr prior) to estimate Watersipora subtorquata abundance (percent cover) in the marina from 2008 to 2009. The long-term panels included 2 racks of 8 panels (each $10 \times 10 \mathrm{~cm}$ ), deployed face down at $1 \mathrm{~m}$ depth. The 2 racks were separated by $\sim 300 \mathrm{~m}$ and showed similar patterns of fluctuation in species' abundances; we thus consider them to be adequate measures of broad marina-wide temporal trends in W. subtorquata abundance. We standardized our time series of raw $W$. subtorquata settlement rate (settlers $\mathrm{cm}^{-2}$ unit $^{-1}$ time) by abundance from the longterm panels to get a 'per capita' settlement rate with units of settlers $\mathrm{cm}^{-2}$ time $\mathrm{e}^{-1}$, per fraction of space occupied. When abundance on the long-term panels was not measured concurrently with our measurement of settlement rates, abundance was interpolated from the 2 nearest points in the time series, allowing us to have estimates of $W$. subtorquata percent cover in the marina corresponding to each of our estimates of settlement rate. This method of standardization is reasonable because $W$. subtorquata disperses for a short enough duration (typically $<8 \mathrm{~h}$ in the lab; Marshall \& Keough 2003) that settling larvae likely come almost entirely from the surrounding marina.

In order to measure the strength of competition during each temporal replicate, for each of the 8 replicate panels, we calculated the percent cover occupied by Watersipora subtorquata at $14 \mathrm{wk}$, divided by the number of $W$. subtorquata settlers present on that plate at $3 \mathrm{wk}$. This quantity estimates fitness per settler in terms of the percent cover of successful recruitment, per settler initially present. We thereby combined both survival and the growth of survivors into 1 measure of fitness (hereafter 'per capita recruitment'). We used this quantity to measure the strength of competition during recruitment as

$$
P(t)=1-\frac{R_{o}(t)}{R_{\mathrm{e}}(t)}
$$

where $R(t)_{o}$ is the observed per capita recruitment at time $t, R_{\mathrm{e}}(t)$ is the per capita recruitment expected in the absence of competition at time $t$, and $P(t)$ is the proportion of potential recruitment lost to competition from other settlers at time $t$, ranging from 0 to 1 . The expected per capita recruitment in the absence of competition was estimated using the W. subtorquata monoculture panels deployed at the same time (Appendix 1). We also used the amount of free space as a component of competition, because the amount of settlement at one time may affect the amount of free space available for settlement at later times. Free space was estimated using the same long-term panels used to measure adult $W$. subtorquata abundance. The full competitive effect was then calculated as

$$
C(t)=P(t)(1-F(t))
$$

where $F(t)$ is the proportion of free space in the marina at time $t$. Thus $C(t)$ is the proportion of potential recruitment lost due to both competition from other settlers, as well as from the preemption of space by prior recruits. $C(t)$ ranges from 0 to 1 , with 0 being no competition and 1 being total loss of recruitment to competition. We hereafter refer to this quantity as the 'competitive effect' during a time period.

Our measures of per capita settlement and competitive effect both include the number of settlers present at $3 \mathrm{wk}$. This allows for a potential spurious correlation between settlement and competition, if there is measurement error in the number of settlers. However, Watersipora subtorquata settlers are conspicuous (bright red) and countable with the naked eye, and we therefore consider measurement error in that quantity to be negligible. We also asked whether our estimates of per capita settlement and competitive effect are sensitive to error in the estimation of marina-wide abundance of $W$. subtorquata and free space. We found that the results reported here were qualitatively the same regardless of whether or not $W$. subtorquata settler abundance was standardized by marina-wide $W$. subtorquata abundance, and whether or not the amount of free space was included in the measure of the competitive effect. We 
therefore consider these results robust to the details of measuring variation in settlement and competitive effect.

\section{Analysis}

A number of approaches have been used to test for the presence of the storage effect (Cáceres 1997, Adler et al. 2006, Sears \& Chesson 2007, Angert et al. 2009). Similar to the approach of Sears \& Chesson (2007), the approach we took here focuses on quantifying the relationship between the temporal pattern of Watersipora subtorquata settlement, and the temporal pattern of competition experienced by W. subtorquata settlers during recruitment. In the framework of Chesson (2008), W. subtorquata per capita settlement is a component of the time-varying environmental response, and the competitive effect is a component of the time-varying competitive response. In this framework, the environmental response is variation in per capita fitness due to environmental factors other than the density of competitors, while the competitive response is the decrease in fitness due to competitors. Because the storage effect has been thoroughly explored for lottery models and other models of sessile iteroparous organisms (Chesson 1994, 2003, 2008), we refer to these sources for a description of how the storage effect is determined by environment-competition covariance. To relate this study to these theoretical results, we make the assumptions that variation in per capita settlement causes variation in the environmental response, and that variation in the competition experienced by settlers causes variation in the competitive response.

The ability of a species to invade a community of competitors is a function of the relationship between the environmental response and the competitive response, when the species is at low density and competition is dominated by interspecific interactions (Chesson 2008). For Watersipora subtorquata, we quantified this relationship by measuring the correlation between $W$. subtorquata settlement and the competitive effect experienced by $W$. subtorquata settlers. If these 2 quantities are perfectly positively correlated, then an increase in $W$. subtorquata per capita settlement is always met by a proportional increase in competition from heterospecific settlers. For example, if per capita settlement increases 2-fold over a certain period, but the effect of competition increases 2-fold as well (resulting, e.g. in twice as much mortality), then the total amount of new $W$. subtorquata recruitment will be constant over time. In this scenario, temporal variation in settlement and competition do not reduce the overall effect of interspecific competition over time. In contrast, if $W$. subtorquata settlement and the competitive effect are uncorrelated or negatively correlated, then there will be periods when $W$. subtorquata settlement is relatively high but competition is relatively weak. These periods will result in large gains in $W$. subtorquata recruitment, and these gains will persist in the population due to the longevity of $W$. subtorquata colonies. The beneficial effect of settlement fluctuation is therefore a function of the degree of decoupling between $W$. subtorquata settlement and the competitive effect experienced by those settlers. In this system, fluctuation in competition is likely driven by fluctuation in the settlement of competing species, and therefore coexistence will be promoted by differentiation of competitors' settlement patterns. However, here we quantify the effect of competition directly, and therefore we directly address whether $W$. subtorquata tends to have abundant settlement when competition is weak.

The expectations described above assume that Watersipora subtorquata is at low density. As W. subtorquata density increases, intraspecific competition will increase, and will be greatest during high settlement periods. This will in turn make the settlementcompetition correlation more positive. For this study it is reasonable to consider $W$. subtorquata to be at effectively low density, because $W$. subtorquata is such a poor competitor during recruitment that all the competition it experiences is due to overgrowth by heterospecifics (K. Edwards pers. obs.), and accordingly $W$. subtorquata settlement rate does not predict $W$. subtorquata per capita recruitment (i.e. there is no evidence of intraspecific effects during recruitment; see 'Results'). Furthermore, W. subtorquata is at low enough abundance marina-wide $(\sim 10 \%$ cover $)$ that it should not greatly alter the densities of competing species, compared to what their abundances would be if $W$. subtorquata were truly invading at very low density. We therefore consider ambient conditions to be a reasonable approximation of 'invader' conditions for $W$. subtorquata, in the context of competition during recruitment.

In order to investigate the mechanism behind temporal variation in the competitive effect, we used regression to relate Watersipora subtorquata per capita recruitment to the settlement rate of abundant competing species, as well as W. subtorquata itself. As predictors we used the solitary ascidian Ascidia ceratodes, which has been shown in prior work to be a very strong competitor during recruitment (Ed- 
wards \& Stachowicz 2011), as well as 3 colonial ascidians that frequently preempt space and sometimes overgrow W. subtorquata settlers (Botrylloides violaceus, Botryllus schlosseri, and Diplosoma listerianum). Observations indicate that the majority of the competitive effect on $W$. subtorquata is due to these 4 species, because they have high settlement rates and are of moderate to strong competitive ability (Edwards \& Stachowicz 2011). In order to reduce the number of collinear variables in the model, we summed the settlement rates of the 3 colonial ascidians to create 1 'colonial ascidian' variable. We used a generalized linear model to test the relationship between $W$. subtorquata per capita recruitment and the settlement rates of $A$. ceratodes, the colonial ascidians, and W. subtorquata itself (using R 2.11.0; $\mathrm{R}$ Development Core Team 2010). Because the response variable is continuous and by definition $\geq 0$, we used a Tweedie distribution with log link (Dunn 2009). For this analysis we used each replicate plate from all temporal replicates $(n=143)$, which allowed us to take advantage of any within-period spatial variation to better estimate the per capita competitive effects of the predictor species.

\section{RESULTS}

Watersipora subtorquata per capita settlement rate varied from 0.35 to 6.3 settlers $\mathrm{cm}^{-2} \mathrm{wk}^{-1}$, per fraction of space occupied (Fig. 1; fraction of space $=\%$ cover/100). The effect of competition on W. subtorquata recruitment at $14 \mathrm{wk}$ varied from 0.57 to 0.93 (i.e. W. subtorquata recruitment was 57 to $93 \%$ less than that expected without competition; Fig. 1). Across both years, W. subtorquata per capita settle- ment and the effect of competition were uncorrelated (Fig. 1C; Pearson's $\mathrm{r}=0.10, \mathrm{n}=20$, bootstrap $95 \%$ $\mathrm{CI}=-0.31$ to 0.51 ). For 2008 alone, W. subtorquata per capita settlement and the effect of competition were uncorrelated (Fig. 1A, C; Pearson's r = -0.32, n = 10 , bootstrap $95 \% \mathrm{CI}=-0.92$ to 0.57 ). For 2009 alone, per capita settlement and the strength of competition were positively correlated (Fig. $1 \mathrm{~B}, \mathrm{C} ; \mathrm{r}=0.78, \mathrm{n}=10$, bootstrap $95 \% \mathrm{CI}=0.31$ to 0.98 ). When comparing yearly means for 2008 and 2009, mean per capita settlement was higher in 2009 (3.79 versus 1.85 settlers $\mathrm{cm}^{-2} \mathrm{wk}^{-1}$ ), while the mean effect of competition was slightly lower in 2009 (0.73 versus 0.79).

Per capita recruitment of Watersipora subtorquata is predicted both by the settlement rate of the solitary ascidian Ascidia ceratodes and by the total settlement rate of the colonial ascidians (likelihood ratio $\chi^{2}$ for $A$. ceratodes: 36.0, $\mathrm{p}<0.001$; for colonials: 10.1 , $\mathrm{p}=0.002$; Table 1, Fig. 2). W. subtorquata settlement rate did not predict per capita recruitment, indicating no evidence for intraspecific competition (likelihood ratio $\chi^{2}=0.11, \mathrm{p}=0.74$; Table 1 , Fig. 2). The per capita effect of $A$. ceratodes was $\sim 7$ times greater than the per capita effect of the colonial ascidians (respective coefficients \pm 1 SE: $-5.7 \times 10^{-2} \pm 5.6 \times$ $10^{-3},-8.7 \times 10^{-3} \pm 2.4 \times 10^{-3}$; Table 1$)$. Average settlement rate of $A$. ceratodes was about 5 times greater in 2008 compared to 2009 (Fig. 3A,B; respective means of 6.4 and 1.3 settlers $100 \mathrm{~cm}^{-2} \mathrm{wk}^{-1}$ ). Summed settlement of 3 common colonial ascidians was similar in both years (Fig. 3C,D; respective means of 17 and 18 settlers $100 \mathrm{~cm}^{-2} \mathrm{wk}^{-1}$ ). The temporal pattern of $A$. ceratodes settlement across both years combined was uncorrelated with the temporal pattern of W. subtorquata per capita settlement $(\mathrm{r}=-0.05, \mathrm{n}=$ 20 , bootstrap $95 \% \mathrm{CI}=-0.41$ to 0.32 ). The temporal
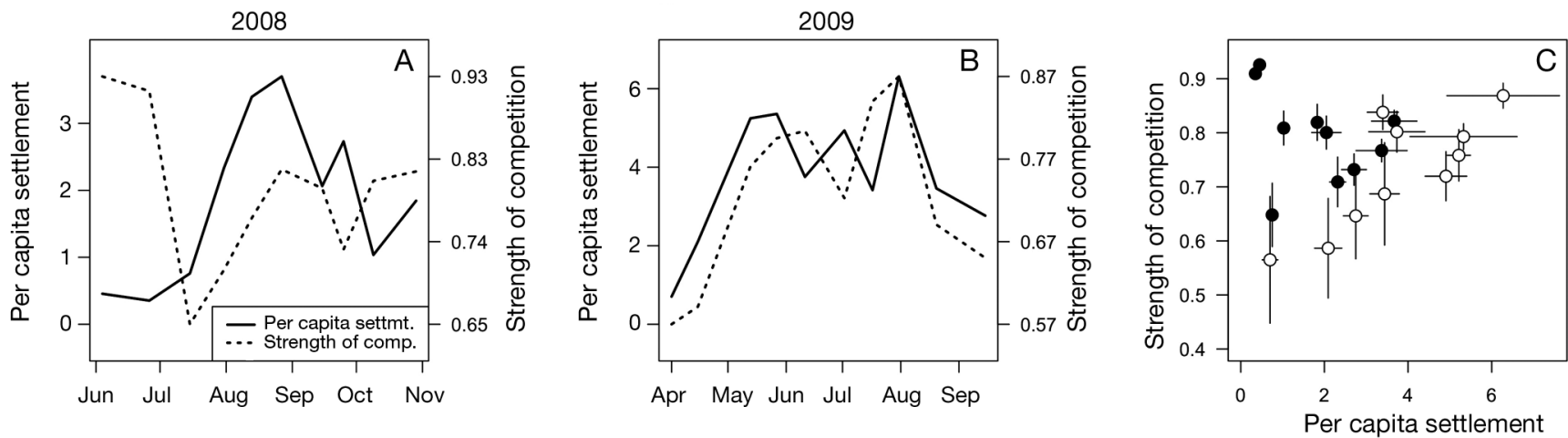

Fig. 1. Watersipora subtorquata. Relationship between settlement and competitive effect during 2008 to 2009 . (A) Time series of mean per capita settlement (solid line) and mean competitive effect (dashed line) during 2008. Units for these quantities are described in 'Materials and methods: Quantifying settlement and competition'. (B) Time series of mean per capita settlement (solid line) and mean competitive effect (dashed line) during 2009. (C) The same data as in (A) and (B), presented as a scatterplot with bootstrap standard errors. (•) Data from 2008: (O) data from 2009 
Table 1. Watersipora subtorquata. Generalized linear model of per capita recruitment as a function of settlement densities of competitors. An interaction between the solitary ascidian $A$. ceratodes and the colonial ascidians was included to account for mutual competitive effects. Interactions involving the Watersipora term were nonsignificant and were dropped from the model. The model was fit using a Tweedie distribution (with index parameter 1.5) and log link. Other index parameters for the Tweedie distribution gave very similar results

\begin{tabular}{|lccrc|}
\hline Term & $\begin{array}{c}\text { Likelihood } \\
\text { ratio }\left(\chi^{2}\right)\end{array}$ & $\mathrm{p}$ & Coefficient & SE \\
\hline Watersipora & 0.02 & 0.90 & $1.1 \times 10^{-3}$ & $6.9 \times 10^{-3}$ \\
Ascidia & 22.0 & $2.7 \times 10^{-6}$ & $-5.4 \times 10^{-2}$ & $7.7 \times 10^{-3}$ \\
Colonials & 8.0 & $4.6 \times 10^{-3}$ & $-8.5 \times 10^{-3}$ & $2.5 \times 10^{-3}$ \\
Ascidia $\times$ Colonials & 17.6 & $2.7 \times 10^{-5}$ & $5.1 \times 10^{-4}$ & $9.2 \times 10^{-5}$ \\
\hline
\end{tabular}

pattern of settlement of the colonial ascidians across both years combined was positively correlated with W. subtorquata per capita settlement $(\mathrm{r}=0.52, \mathrm{n}=20$, bootstrap $95 \% \mathrm{CI}=0.23$ to 0.81 ).

\section{DISCUSSION}

Our results demonstrate that over 2008 to 2009, Watersipora subtorquata per capita settlement was uncorrelated with the competitive effect experienced by $W$. subtorquata settlers. However, the 2 years exhibited distinct patterns, and on a within-year basis, settlement and competition were uncorrelated in 2008, but positively correlated in 2009 (Fig. 1). On an across-year basis, the year with higher mean settlement (2009) had a lower mean competitive effect. Accordingly, storage effect theory predicts that combined intra- and interannual settlement fluctuation could have benefited $W$. subtorquata persistence, and intra-annual fluctuation would be most beneficial in 2008, while in 2009 the effect would have been weak at best. An intuitive understanding of this difference can be gleaned from the time series plotted in Fig. 1A,B. In 2008, there were periods where $W$. subtorquata settlement was high but competition was low or moderate (e.g. August), and periods where competition was high but settlement was low (e.g. June). Periods when settlement is high but competition is relatively low lead to increased recruitment of adults (Fig. 2, Table 1). In contrast, in 2009, periods of high settlement were strongly correlated with periods of high competition (e.g.

August), and vice versa (e.g. April).

Some mechanistic understanding of the difference between 2008 and 2009 can be gained from a comparison of the settlement of the main competitors of Watersipora subtorquata in the 2 years. Settlement rate of the solitary ascidian Ascidia ceratodes differed greatly between 2008 and 2009, with only onefifth as much settlement in 2009 on average (Fig. 3). In contrast, the mean settlement rate of the colonial ascidians was nearly identical in the 2 years (Fig. 3). A. ceratodes is a very strong competitor versus Watersipora (Edwards \& Stachowicz 2011; Table 1), and in addition it tends to have a settlement pattern that is uncorrelated with $W$. subtorquata settlement. In contrast, the colonial ascidians have a significant but weaker per capita effect on $W$. subtorquata, and their pattern of settlement tends to be positively correlated with $W$. subtorquata settlement. The combination of these observations suggests that in 2008, temporal variation in competition is driven by temporal variation in $A$. ceratodes settlement, and this variation in competition is uncorrelated with $W$. subtor-
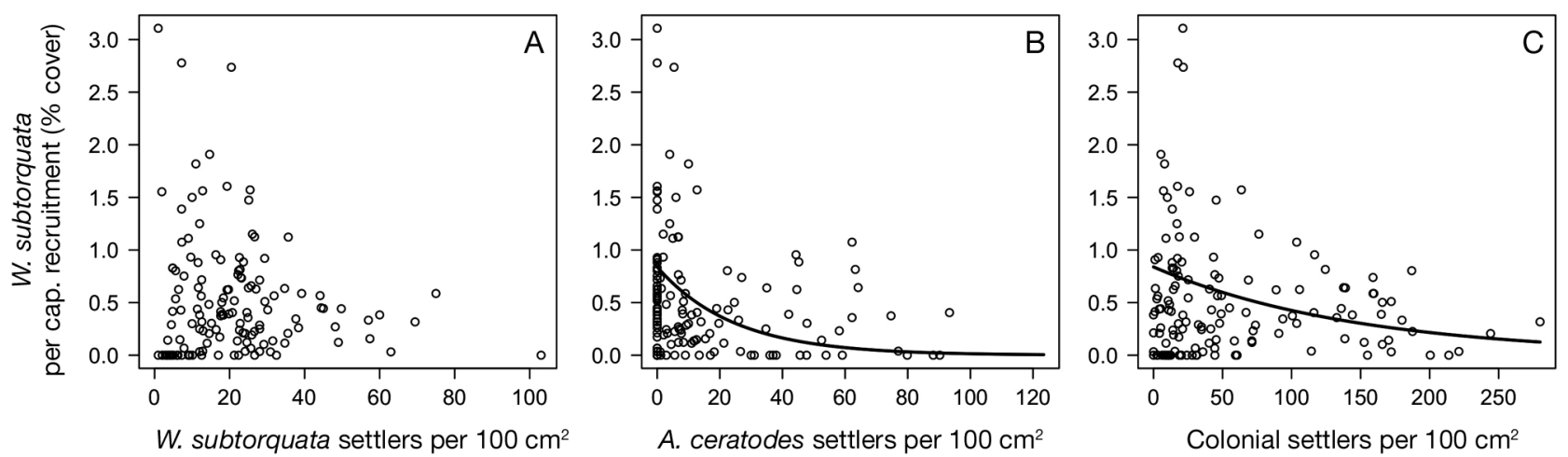

Fig. 2. Watersipora subtorquata. Effect of settler density at 3 wk of (A) W. subtorquata, (B) Ascidia ceratodes, and (C) colonial ascidians on recruitment of Watersipora. Points are from individual experimental panels, for 2008 and 2009 . Lines are generalized linear model fits (Table 1). Watersipora per capita recruitment was calculated as percent cover at 14 wk, divided by the number of settlers present at $4 \mathrm{wk}$ 


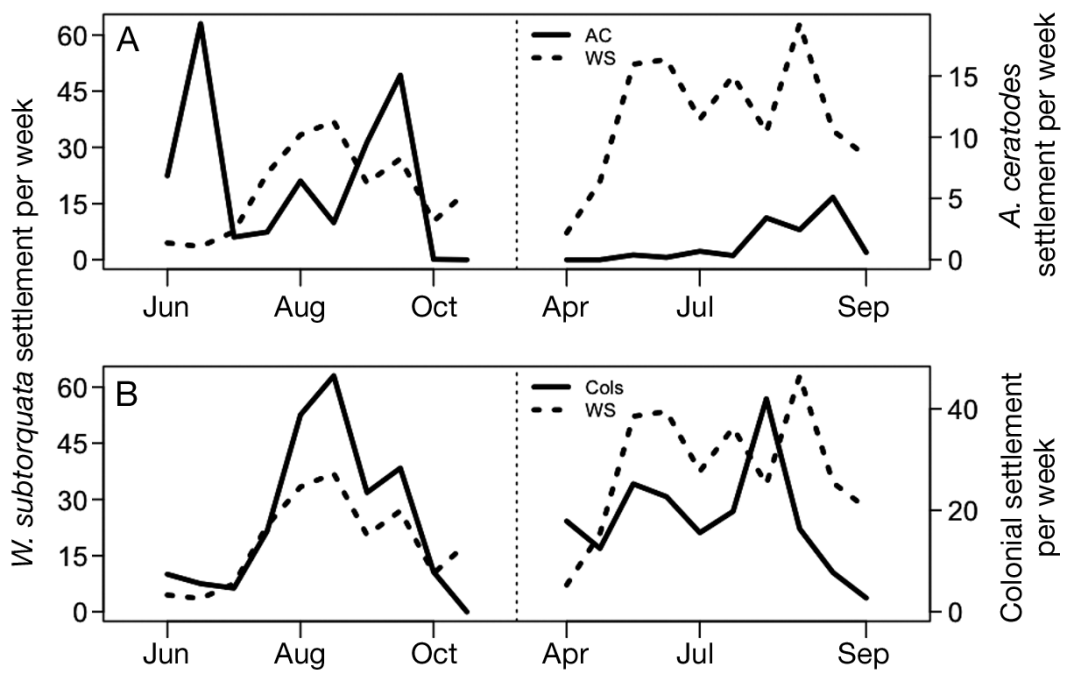

Fig. 3. Patterns of settlement for Watersipora subtorquata (WS), Ascidia ceratodes (AC), and the colonial ascidians (Cols) during 2008 and 2009. Settlement rate for $W$. subtorquata is the per capita estimate described in 'Materials and methods: Quantifying settlement and competition'. Settlement rate for $A$. ceratodes and the colonial ascidians is in settlers $100 \mathrm{~cm}^{-2} \mathrm{wk}^{-1}$. (A) Comparison of settlement for Watersipora (dashed line) and A. ceratodes (solid line) in 2008 (left panel) and 2009 (right panel). (B) Comparison of settlement for W. subtorquata (dashed line) and the colonial ascidians (solid line) in 2008 (left panel) and 2009 (right panel)

quata settlement because the species have different settlement phenologies. In contrast, in 2009 A. ceratodes was too rare to drive competitive effects, and therefore temporal variation in competition was driven by the colonial ascidians, which have a similar phenology to $W$. subtorquata. This interpretation is supported by direct field observations, which indicated that periods of peak Ascidia settlement in 2008 had nearly $100 \%$ dominance of the substrate by A. ceratodes at recruitment. In contrast, this never occurred during 2009, and throughout the year the main spaceholders were the colonial ascidians (K. Edwards pers. obs.).

Our results add to the mounting evidence that species-specific responses to environmental variation can promote coexistence via the storage effect (Cáceres 1997, Adler et al. 2006, Angert et al. 2009). The focus of prior work has been on interannual variation in recruitment or growth; our study suggests that for organisms with continuously reproducing populations and relatively short 'juvenile' periods, intra-annual variability may promote coexistence as well. Our results also suggest that the importance of the storage effect as a coexistence mechanism may itself be temporally variable. In this system, the longterm importance of the mechanism will depend upon the frequency of years in which Ascidia ceratodes is common or rare during recruitment. Although we lack long-term data on A. ceratodes recruitment, we observed dominance of A. ceratodes in 2007 that was similar to its effects in 2008. For competitors with similar recruitment phenologies (e.g. Watersipora subtorquata and the colonial ascidians), coexistence is likely maintained by other mechanisms, such as a colonization-competition tradeoff (Edwards \& Stachowicz 2010).

Early work on the storage effect was motivated by the variability in larval settlement that is typical of benthic marine communities (Sale 1977, Chesson \& Warner 1981). Our work provides evidence for this mechanism in a community of sessile invertebrates, and we expect that the mechanism is important in a wide range of benthic communities. In many communities, newly settling individuals experience strong competitive effects, while established individuals can preempt space or other resources from competitors (reef fish: Geange \& Stier 2009; mussels and barnacles: Wootton 1993; corals: reviewed by Connolly \& Muko 2003). This kind of size- or age-dependent competitive interaction, in combination with species-specific temporal patterns of larval settlement patterns (e.g. Sutherland \& Karlson 1977, Wallace 1985, Hurlbut 1991), has the potential to contribute significantly to coexistence, and may help explain the great species diversity of the benthic organisms that compete for a small number of limiting resources.

Prior work in this system has shown that spatially stochastic settlement promotes the persistence of Watersipora subtorquata, particularly in its interaction with Ascidia ceratodes (Edwards \& Stachowicz 2011). Although this work was not framed in terms of the storage effect, the mechanism by which spatially stochastic settlement promotes coexistence can be considered a spatiotemporal analogue of the purely temporal storage effect described here (Chesson 2000, Berkley et al. 2010). We consider it likely that for this community and benthic communities in general, spatial and temporal settlement variability act jointly to promote species coexistence.

In this study we focused on competition for free patches of substrate, but in this system some species also serve as secondary substrate for other species. Watersipora subtorquata (and other species) can settle on the tunics of Ascidia ceratodes, although $W$. subtorquata experience reduced growth compared to 
growth on primary substrate (Claar et al. 2011). This interaction may ameliorate the strong negative effect of Ascidia on Watersipora, although the benefit to Watersipora will depend on competitive interactions with other epibionts. In addition, Watersipora (and other species) can also settle on the dead older portions of Watersipora colonies; this interaction may amplify the storage effect by increasing the benefit to Watersipora of temporally favorable recruitment periods. Therefore, a full accounting of the maintenance of diversity in this system will require joint consideration of life history tradeoffs (Edwards \& Stachowicz 2010), spatially variable settlement (Edwards \& Stachowicz 2011), temporally variable settlement, the facilitative effects of epibiotic growth (Claar et al. 2011), and likely other untested mechanisms.

Acknowledgements. We thank M. Ryan, O. Schmitz, and 3 anonymous reviewers for comments on the manuscript. Funding was provided by the National Science Foundation through a Graduate Research Fellowship to K.F.E. and grants to J.J.S. from the Biological Oceanography Program (OCE 03-51778 and 08-50707).

\section{LITERATURE CITED}

Adler PB, HilleRisLambers J, Kyriakidis PC, Guan QF, Levine JM (2006) Climate variability has a stabilizing effect on the coexistence of prairie grasses. Proc Natl Acad Sci USA 103:12793-12798

Angert AL, Huxman TE, Chesson P, Venable DL (2009) Functional tradeoffs determine species coexistence via the storage effect. Proc Natl Acad Sci USA 106: 11641-11645

Berkley HA, Kendall BE, Mitarai S, Siegel DA (2010) Turbulent dispersal promotes species coexistence. Ecol Lett 13: 360-371

Berlow EL (1999) Strong effects of weak interactions in ecological communities. Nature 398:330-334

> Cáceres CE (1997) Temporal variation, dormancy, and coexistence: a field test of the storage effect. Proc Natl Acad Sci USA 94:9171-9175

Caley MJ, Carr MH, Hixon MA, Hughes TP, Jones GP, Menge BA (1996) Recruitment and the local dynamics of open marine populations. Annu Rev Ecol Syst 27: 477-500

> Chesson P (1994) Multispecies competition in variable environments. Theor Popul Biol 45:227-276

Chesson PL (2000) General theory of competitive coexistence in spatially-varying environments. Theor Popul Biol 58:211-237

Chesson P (2003) Quantifying and testing coexistence mechanisms arising from recruitment fluctuations. Theor Popul Biol 64:345-357

Chesson P (2008) Quantifying and testing species coexistence mechanisms. In: Valladers F, Camacho A, Elosegui A, Gracia C, Estrada M, Senar JC, Gili JM (eds) Unity in diversity: reflections on ecology after the legacy of Ramon Margalef. Fundacion BBVA, Bilbao, p 119-164
Chesson PL, Warner RR (1981) Environmental variability promotes coexistence in lottery competitive systems. Am Nat 117:923-943

> Claar DC, Edwards KF, Stachowicz JJ (2011) Positive and negative effects of a dominant competitor on the settlement, growth, and survival of competing species in an epibenthic community. J Exp Mar Biol Ecol 399: 130-134

> Connolly SR, Muko S (2003) Space preemption, size-dependent competition, and the coexistence of clonal growth forms. Ecology 84:2979-2988

Dunn PK (2009) Tweedie: Tweedie exponential family models. R package version 2.0.2. http://cran.r-project.org/ web/packages/tweedie/index.html

> Edwards KF, Stachowicz JJ (2010) Multivariate trade-offs, succession, and phenological differentiation in a guild of colonial invertebrates. Ecology 91:3146-3152

Edwards KF, Stachowicz JJ (2011) Spatially stochastic settlement and the coexistence of benthic marine animals. Ecology 92:1094-1103

Gaines S, Roughgarden J (1985) Larval settlement rate: a leading determinant of structure in an ecological community of the marine intertidal zone. Proc Natl Acad Sci USA 82:3707-3711

> Geange SW, Stier AC (2009) Order of arrival affects competition in two reef fishes. Ecology 90:2868-2878

> Hurlbut CJ (1991) Community recruitment: settlement and juvenile survival of seven co-occurring species of sessile marine invertebrates. Mar Biol 109:507-515

Kelly CK, Bowler MG (2002) Coexistence and relative abundance in forest trees. Nature 417:437-440

> Marshall DJ, Keough MJ (2003) Variation in the dispersal potential of non-feeding invertebrate larvae: the desperate larva hypothesis and larval size. Mar Ecol Prog Ser 255:145-153

- Marshall DJ, Keough MJ (2009) Does interspecific competition affect offspring provisioning? Ecology 90:487-495

> Pake CE, Venable DL (1995) Is coexistence of Sonoran Desert annuals mediated by temporal variability in reproductive success? Ecology 76:246-261

R Development Core Team (2010) R: a language and environment for statistical computing. R Foundation for Statistical Computing, Vienna

Sale PF (1977) Maintenance of high diversity in coral reef fish communities. Am Nat 111:337-359

Sears ALW, Chesson P (2007) New methods for quantifying the spatial storage effect: an illustration with desert annuals. Ecology 88:2240-2247

Sutherland JP, Karlson RH (1977) Development and stability of the fouling community at Beaufort, North Carolina. Ecol Monogr 47:425-446

Underwood AR, Keough MJ (2000) Supply-side ecology: the nature and consequences of variations in recruitment of intertidal organisms. In: Bertness MD, Gaines SD, Hay ME (eds) Marine community ecology. Sinauer Associates, Sunderland, MA, p 183-200

Wallace CC (1985) Reproduction, recruitment and fragmentation in nine sympatric species of the coral genus Acropora. Mar Biol 88:217-233

> Warner RR, Chesson PL (1985) Coexistence mediated by recruitment fluctuations: a field guide to the storage effect. Am Nat 125:769-787

> Wootton JT (1993) Size-dependent competition: effects on the dynamic vs. the end point of mussel bed succession. Ecology 74:195-206 
Appendix 1. Estimation of Watersipora subtorquata recruitment in the absence of competition

In order to quantify the reduction in per capita Watersipora subtorquata recruitment due to competition, we estimated the per capita recruitment expected in the absence of competition, using experimental panels in which all species but $W$. subtorquata were removed. We pooled the data from our monoculture panels and fit a saturating exponential curve, $y_{i}=100 \times\left[1-\exp \left(b \times x_{i}\right)\right]$, where $y_{i}$ is the percent cover of $W$. subtorquata on plate $i$ at $14 \mathrm{wk}$, and $x_{i}$ is the number of $W$. subtorquata settlers on plate $i$ at $3 \mathrm{wk}$. This curve estimates recruitment per settler in terms of percent cover, and saturates at $100 \%$ as the number of settlers increases (Fig. A1). At low settler density, this curve is approximately equal to $y_{i}=100 \times b \times x_{i l}$ and therefore $100 \times b$ estimates the percent cover per settler in the absence of competition. We fit this relationship using nonlinear least squares, and the fitted value of $b$ was 3.2 (95\% boostrap confidence interval $=2.8$ to 3.7 ). Although the residuals are not normal over the entire range of the predictor (due to the boundaries at 0 and $100 \%$ cover), the exact value of $b$ does not affect the results described in the main text.

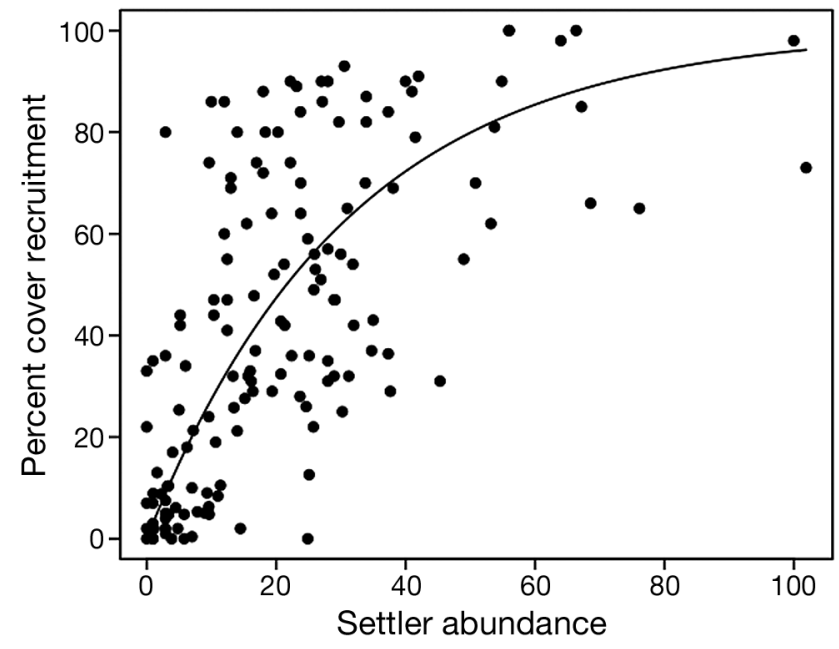

Fig. A1. Watersipora subtorquata. Relationship between recruitment and settler abundance

Editorial responsibility: Roger Hughes,

Bangor, UK
Submitted: January 27, 2012; Accepted: May 16, 2012 Proofs received from author(s): August 8, 2012 